\title{
The Effect of Metformin on Pregnancy Outcome Among Filipino Women with Polycystic Ovary Syndrome
}

\author{
May L. Uyking-Naranjo ${ }^{1}$, Evan Paulo D. Consencino ${ }^{1}$
}

Roberto C. Mirasol ${ }^{1}$, Joan T. Tan-Garcia ${ }^{2}$

${ }^{1}$ Department of Internal Medicine, Section of Endocrinology, Diabetes and Metabolism,

${ }^{2}$ Department of Obstetric and Gynecology, Section of Reproductive Endocrinology and Infertility,

St. Luke's Medical Center, Philippines

\section{BACKGROUND}

The use of metformin in PCOS is increasingly accepted but its therapeutic use during pregnancy is still a debatable issue. There are few local studies on the use of metformin among PCOS patients and data is lacking among Filipinos.

\section{OBJECTIVES}

To determine the effect of metformin on maternal and neonatal outcome among pregnant Filipino women with PCOS.

\section{METHODOLOGY}

DESIGN: Retropsective Cohort Study

- Outcome Measures:

Maternal outcome - rate of first trimester spontaneous abortion, development of gestational diabetes,

pregnancy-induced hypertension, mode of delivery and gestational age of delivery.

Neonatal outcome -live birth rates, APGAR score, infants birth weight and development of congenital anomaly.

Figure. Schematic diagram of the study

337

Treated with metformin : 324

With pregnancy : 165

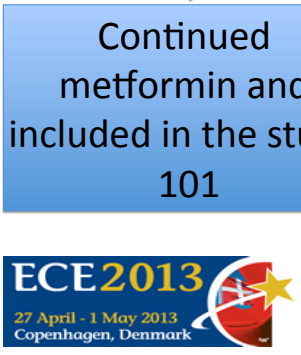

Discontinued metformin : 64 Had no data : 4 Included in the study: 60
Table 1. Maternal outcome among pregnant PCOS

\begin{tabular}{|c|c|c|c|}
\hline & $\begin{array}{c}\text { CONTINUED } \\
\text { METFORMIN(N=1 } \\
01) \\
\text { N(\%) }\end{array}$ & $\begin{array}{c}\text { DISCONTINUED } \\
\text { METFORMIN(N=60) } \\
\text { N(\%) }\end{array}$ & P value \\
\hline $\begin{array}{l}\text { First trimester spontaneous } \\
\text { abortion }\end{array}$ & $5(5.0 \%)$ & $21(36.2 \%)$ & $<0.001$ \\
\hline GDM & $19(19.0 \%)$ & $18(32.6 \%)$ & 0.071 \\
\hline $\begin{array}{l}\text { Pregnancy ind uced- } \\
\text { hypertension }\end{array}$ & 0 & 0 & \\
\hline \begin{tabular}{|l} 
Age of gestation \\
Pre-term \\
Term \\
Post-Term \\
\end{tabular} & $\begin{array}{c}9(9.8 \%) \\
82(89.1 \%) \\
1(1.1 \%)\end{array}$ & $\begin{array}{c}1(2.7 \%) \\
36(97.3 \%) \\
0\end{array}$ & 0.317 \\
\hline $\begin{array}{l}\text { Mode of delivery } \\
\quad \text { NSVD } \\
\text { CS } \\
\end{array}$ & $\begin{array}{l}41(44.6 \%) \\
51(55.4 \%)\end{array}$ & $\begin{array}{l}18(47.4 \%) \\
20(52.6 \%)\end{array}$ & 0.770 \\
\hline
\end{tabular}

Table 2.Multivariate analysis of the effect of metformin during pregnancy on spontaneous abortion

\begin{tabular}{|l|c|c|}
\hline Variable & Odds Ratio (95\% CI) & P value \\
\hline Metformin during pregnancy & $0.168(0.048-0.592)$ & 0.005 \\
\hline Age diagnosed with PCOS & $1.076(0.932-1.241)$ & 0.318 \\
\hline Serum Insulin & $1.099(0.995-1.214)$ & 0.062 \\
\hline
\end{tabular}

Table 3. Neonatal outcome among pregnant PCOS

\begin{tabular}{|l|c|c|c|}
\hline & $\begin{array}{c}\text { CONTINUED } \\
\text { METFORMIN } \\
\text { N(\%) }\end{array}$ & $\begin{array}{c}\text { DISCONTINUED } \\
\text { METFORMIN } \\
\text { N(\%) }\end{array}$ & P value \\
\hline Live birth & $90(97.8 \%)$ & $37(100 \%)$ & 0.366 \\
\hline $\begin{array}{l}\text { Congenital anomaly on } \\
\text { ultrasound }\end{array}$ & $1(1.1 \%)$ & $1(2.6 \%)$ & 0.515 \\
\hline $\begin{array}{l}\text { Congenital anomaly at } \\
\text { birth }\end{array}$ & 0 & 0 & \\
\hline & means \pm SD & means \pm SD & \\
\hline $\begin{array}{l}\text { A P G A R s c o r e }, 5 \\
\text { minutes }\end{array}$ & $8.91 \pm 0.32$ & $8.94 \pm 0.23$ & 0.620 \\
\hline Birth weight (grams) & $2840.97 \pm 385$ & $2941.56 \pm 456$ & 0.242 \\
\hline
\end{tabular}

\section{CONCLUSION}

In women with PCOS, continuous use of metformin during pregnancy reduced the rate of first trimester spontaneous abortion 